\title{
Effects of combining both mobilization and hold-relax technique on the function of post-surgical patients with shoulder adhesive capsulitis
}

\author{
Jongchan Jung ${ }^{a}$, Yijung Chung ${ }^{\mathrm{b}}$ (1) \\ ${ }^{a}$ Department of Physical Therapy, Nalgae Hospital, Seoul, Republic of Korea \\ ${ }^{\mathrm{b}}$ Department of Physical Therapy, College of Health Science and Social Welfare, Sahmyook University, Seoul, Republic of Korea
}

Objective: This study investigated the effects of combining both mobilization and hold-relax (HR) technique on the function of post-surgical patients with shoulder adhesive capsulitis.

Design: Randomized controlled trial.

Methods: Forty-five surgical patients with shoulder adhesive capsulitis participated in this study and were randomly divided into three groups; both mobilization with movement (MWM) and HR technique (HR-MWM) group ( $\mathrm{n}=15$ ), the MWM group ( $\mathrm{n}=15$ ), and control group $(n=15)$. All participants received three different interventions; 1) MWM combined with HR technique in PNF stretching on the shoulder, 2) MWM on the shoulder, 3) general physical therapy and intervention with neither MWM or HR stretching. Pre- and post-intervention, each subject was randomly evaluated for shoulder flexion range of motion (ROM), shoulder flexor muscle strength, Visual Analogue Scale (VAS), and the Korean version of the Shoulder Pain And Disability Index (SPADI). Results: The MWM combined with HR technique group had significant effects on shoulder flexion ROM, shoulder flexor muscle strength, VAS and SPADI compared to the MWM and control group $(p<0.05)$. The MWM group showed a significantly greater increase in shoulder flexion ROM compared to the control group $(p<0.05)$.

Conclusions: These findings suggest that combining both the MWM and HR technique on the shoulder may more effectively improve shoulder function than MWM alone or without MWM\&HR technique. Therefore, combining both the MWM and HR technique is a suggested intervention for increasing function due to shoulder adhesive capsulitis after surgery.

Key Words: Adhesive capsulitis, Hold-relax technique, Mobilization, Post-surgical, Shoulder

\section{Introduction}

Adhesive arthritis of the shoulder causes gradual pain in the shoulder joint and causes loss of active and passive mobility. Clinical syndrome includes pain, limited range of motion (ROM), and muscle weakness caused by non-use [1]. Adhesive arthritis is a self-limiting disease that lasts about 2 years [2], and it is a chronic disease that causes disability for a long time [3]. Although the onset of adhesive capsulitis is unknown, some authors suggest that shoulder joint disorders and shoulder movement disorders are asso- ciated with the soft tissue sticking together and the adherent part of the armpit [4]. The limitation of the shoulder ROM is based on the shortening of soft tissue around the shoulder, and the apparent absence correlation between the limited shoulder ROM and the joint space [5]. The adhered part of the armpit inhibits the movement of the head of the humerus [6]. Treatment methods include surgical treatment and non-surgical treatment. When surgery is required under the diagnosis of a doctor, arthroscopic capsulotomy is typically mentioned [7]. Although surgical treatment is viewed to be effective, but post-adhesive capsulitis surgery shows not on-

Received: 26 May, 2020 Revised: 12 June, 2020 Accepted: 15 June, 2020

Department of Physical Therapy, College of Health Science and Social Welfare, Sahmyook University, 815 Hwarang-ro, Nowon-gu, Seoul 01795 , Republic of Korea Tel: 82-2-3399-1637 Fax: 82-2-3399-1639 E-mail: yijung36@syu.ac.kr

(a) This is an Open-Access article distributed under the terms of the Creative Commons Attribution Non-Commercial License (http://creativecommons.org/licenses/ by-nc/4.0) which permits unrestricted non-commercial use, distribution, and reproduction in any medium, provided the original work is properly cited.

Copyright (๑) 2020 Korean Academy of Physical Therapy Rehabilitation Science 
ly the construction of the shoulder joint capsule, but also the muscle construction [8].

Data supports the use of mobilization as a non-surgical treatment method of obtaining normal elongation and soft tissue of the shoulder joint sac, and manual stretching of the joint sac and soft tissue with the help of mobilization was recommended. Mobilization with movement (MWM) is also used to reduce pain [9-12].

Out of the mulligan mobilization techniques, mulligan provided the MWM technique. It was explained that operation was performed in patients with adhesive arthritis [13]. In addition, proprioceptive neuromuscular facilitation (PNF) is a stretching method used to increase the extensibility of soft tissues [14]. PNF is defined as a method of stimulating and enhancing neuromuscular structures through stimulation of intrinsic receptors [15]. This stretching technique was geared towards reducing the promotion of muscle stretching and increasing inhibition. The PNF stretching technique is divided into two types: a contract-relax-contract (CRC) technique and a hold-relax-contract (HRC) technique [14]. Two methods of PNF stretching techniques are commonly used to enhance ROM. Studies have shown that the $\mathrm{HRC}$ technique is more effective for ROM enhancement compared to the static, HRC, and CRC techniques [16].

In previous studies, many methods have been used in clinical practice to increase the extensibility of soft tissues. In the study comparing the complex articular sac pattern and the method of mediating soft tissue shortening, there was a comparison between the operation and the PNF stretching method, but the comparison between the MWM during the mulligan operation and the HRC technique during the PNF stretching was not studied.

Therefore, in parallel with the HRC technique, the purpose of this study was to investigate for changes in shoulder flexion ROM, shoulder flexion muscle strength, VAS, and Shoulder Pain And Disability Index (SPADI) during exercise with MWM in patients with adhesive capsulitis as well as to propose an efficient and systematic method of treatment.

\section{Methods}

\section{Participants}

The selection criteria were men and women between the ages of 40 to 60 years old who could read, speak, and can understand the research procedure, those who have complained of shoulder pain for at least 3 months, those with limited shoulder joint ROM (bending, widening, external/ internal rotation of the shoulder joint) $[1,17]$, and those who were diagnosed with adhesive capsulitis and had surgery.

Exclusion criteria were those who complained of shoulder pain due to severe trauma, those with multiple fractures of the shoulder, those with rotator cuff tears, and those with calcification tendinitis [17]. This study was reviewed and approved by the Institutional Review Board of Sahmyook University (IRB No. SYUIRB 2015-081). A flow chart depicting the patient analysis procedure is presented in Figure 1. One physical therapist with over 10 years of experience in the treatment of shoulder joint surgery was evaluated using a single blind method without knowing about the group of each patient. Shoulder flexor muscle strength, VAS, and SPADI were evaluated. Hold relax (HR)+MWM group combined HRC technique with MWMintervention for 6 times, 15 minutes/ day, 3 times/week for shoulder flexion and external rotation, 4 weeks and 15 times for MWM, 3 sets/day, 3 times/week, 12 times in 4 weeks, the MWM group performed MWM 15 times, 3 sets/day, 3 times/week, 12 times in total for 4 weeks, and the control group performed general intervention in the hospital for 30 minutes/day, 3 times/wk for $4 \mathrm{wks}$, totaling up to 12 times.

\section{Hold relax+mobilization with movement group}

\section{MWM technique}

As a method of producing active joint movement with mobilization for shoulder flexion, the subject was seated in a comfortable sitting position with a belt on the head of the humerus, and the therapist's hand was wrapped around an appropriate area of the humeral head to perform a glide.

Counterpressure was applied onto the scapula by the other hand of the therapist. A glide was applied over the pain-free range during slow active shoulder movements and was released after returning to the starting position. This process was repeated 15 times in 3 sets, and a 1-minute rest period was provided between each set (Figure 2) [18]. With the MWM's method for external rotation of the shoulder joint, the subject had a folded towel under the scapula while lying in a comfortable position, and the elbow flexed to $90^{\circ}$. The subject held the stick with both hands, and then pushed the stick with the opposite hand to produce shoulder external rotation. In the meantime, the therapist stood on the other side and held the subject's humeral head and applied the slide within pain-free range, back and outwards based on the joint concavity. This process was repeated 15 times in 3 sets, and a 30 seconds rest period was provided between each set 

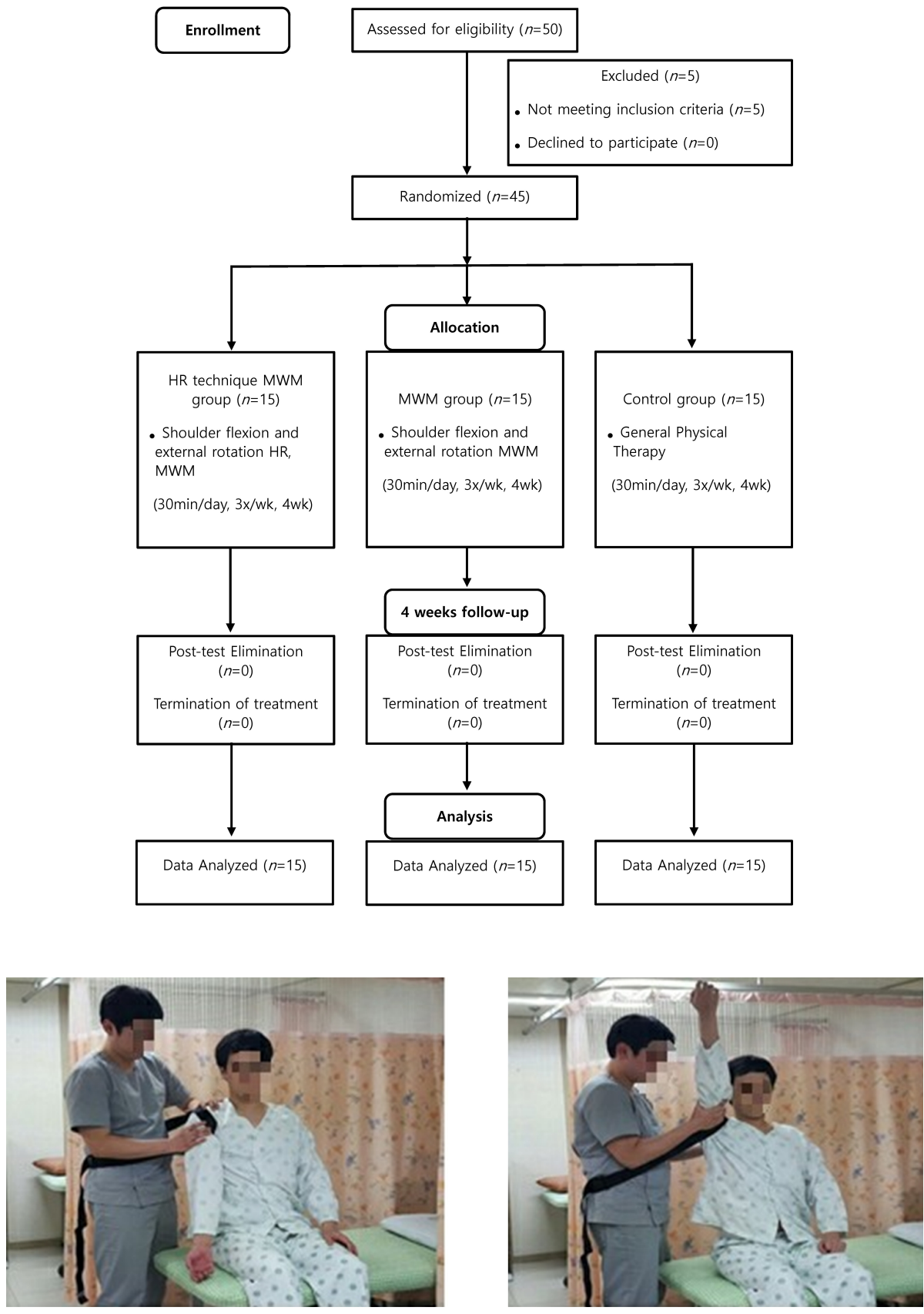

Figure 2. MWM for shoulder flexion. MWM: mobilization with movement.
(Figure 3) [18]. A pressure gauge (hand dynamomter) was used to apply a uniform pressure $(5 \mathrm{~N})$ to the slide that was applied to the subject [19].

\section{HR technique}

The HR technique was applied in supine position, and the shoulder joint was slightly relaxed at the end of the flexion range up to where the patient felt pain, so the therapist slowly provided resistance in the direction of flexion, abduction, or external rotation, and without the subject intending to move, the subject tried to go against the resistance towards extension, adduction, and internal rotation, with particular emphasis on trying to resist external rotation.

The therapist's bare-hand was placed in contact onto the antagonist muscle area to apply resistance and gradually increased the resistance by isometric contraction to reach the maximum resistance. When relaxation was achieved by inducing relaxation, this procedure was repeated 6 times (Figures 4, 5).

Then, after securing the newly obtained range, it was re- 

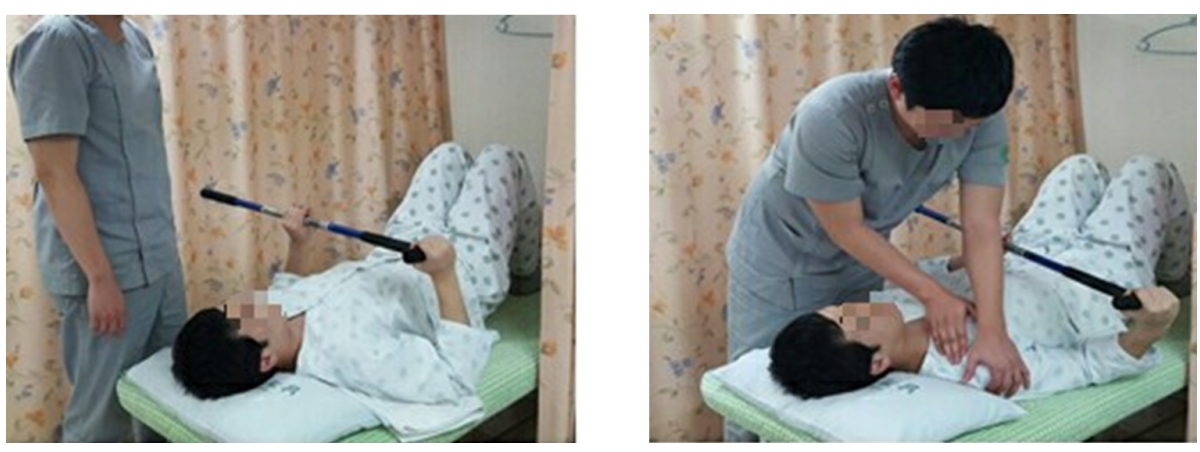

Figure 3. MWM for shoulder external rotation. MWM: mobilization with movement.
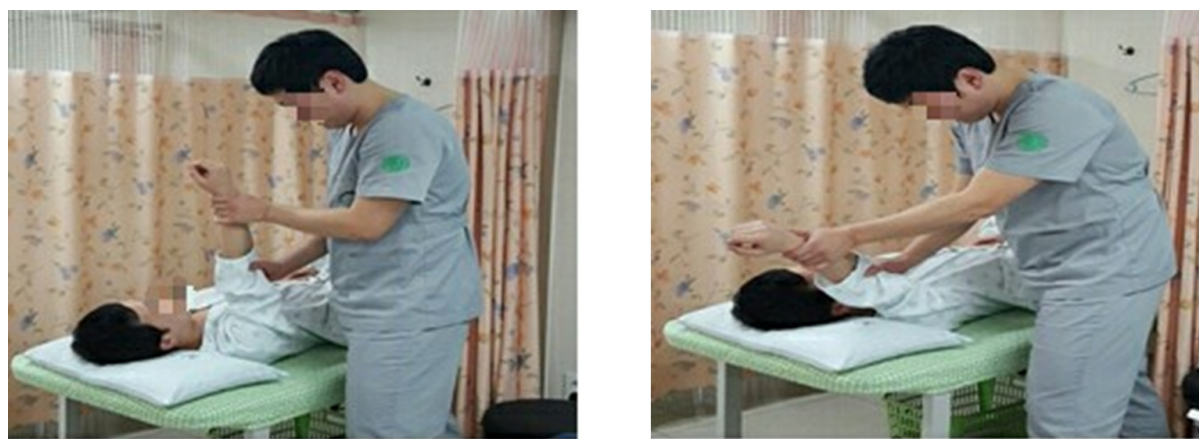

Figure 4. HR PNF stretching for shoulder flexion. HR: hold-relax, PNF: proprioceptive neuromuscular facilitation.
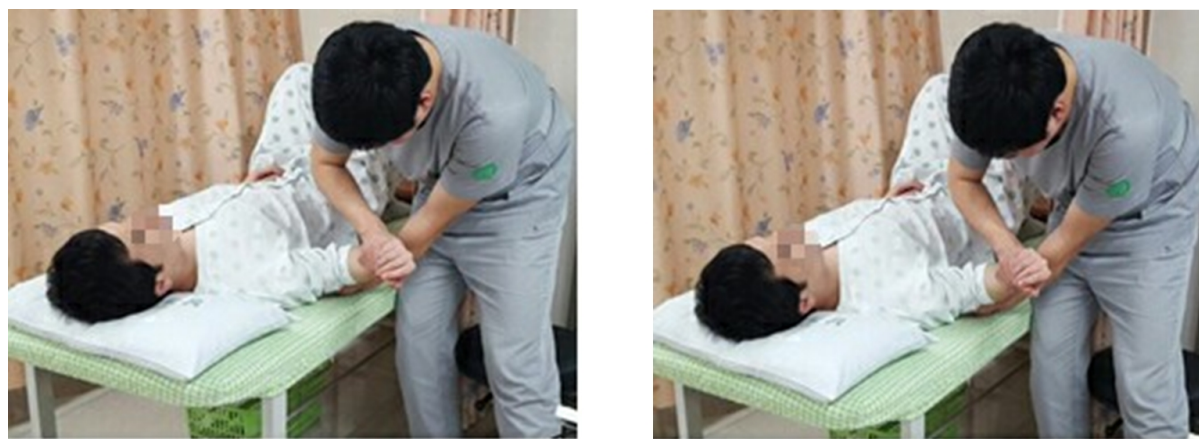

Figure 5. HR PNF stretching for shoulder external rotation. HR: holdrelax, PNF: proprioceptive neuromuscular facilitation.

peatedly attempted again at the limit point and carried out for a total of 15 minutes [20].

\section{Mobilization with movement group}

The MWM technique of the HR-MWM group was applied to the MWM group.

\section{Control group}

Patients with adhesive capsulitis surgery were instructed to minimize active movements with their arms on the splint on a continuous passive motion (CPM) for shoulder joint flexion while facing the front. Patients performed passive exercise and increased the range just before the maximum pain was felt for each exercise [21].

All subjects were treated for 30 minutes after intervention and received CPM during the remaining time. The control group was also received CPM for 30 minutes.

\section{Outcome measurement}

In order to measure the shoulder flexion ROM, the subject maintained the anatomical posture in the supine position, and a goniometer was used to measure the flexion angle of the humeral head with the axis of the shoulder joint [18].

Maximum shoulder flexion strength was measured by maintaining the muscle power at $90^{\circ}$ of shoulder flexion with a palm-up position against vertically applied resistance for 5 seconds using the Nicholas frequency muscle strength evaluation tool (Lafayette, IN, USA) [22]. Each condition was repeatedly measured three times, and the experiment was conducted after having subjects take a 1-minute break between the conditions. The measurement method was conducted in a random order, and for the subjects' safety, one as- 
sistant was present to assist with the experiment. The test-retest isometric muscle test reliability of digital Nicholas frequency muscle strength evaluation is 0.91 to 0.99 [23].

A hand dynamometer (Sammons Preston, Inc., Bolingbrook, IL, USA) was used to measure the amount of pressure applied to the MWM slide provided by the therapist to shoulder joint of the subject [19]. The reliability of the hand dynamometer is $0.85-0.98$ [24].

The VAS was used to evaluate shoulder joint pain. The subject was presented a $100 \mathrm{~mm}$ scale-free line and the subject was instrcuted to make a mark on the line to according to their level of pain. The score was set to 0 in the absence of pain, and to 100 for pain that was considered unbearable [25]. The inter-rater test-retest reliability of the Visual Analog Scale is 0.97 [26].

To evaluate the degree of shoulder dysfunction, the Korean version of the SPADI was used, which was ompleted in the form of a questionnaire, and it was divided into two subscales: five pain subscale questions and eight function/ disability subscale questions. Each individual item carries the same within each area, and the score of the area is converted into percentage $(\%)$ where 0 represents perfect condition and 100 represents the worst condition. The total score for this tool is determined by averaging the scores of 13 evaluation items.

The criterion-related validity was examined by correlating the results of the numerical rating scale (NRS), disability of the arm, shoulder and hand (DASH), and ROM scores [27]. The inter-rater test-retest reliabiilty of the SPADI is 0.99, and the internal consistency was Cronbach alpha was 0.942. SPADI was significantly correlated with NRS $(\mathrm{r}=$ $0.946)$, DASH $(\mathrm{r}=0.935)$, and ROM $(\mathrm{r}=0.927)$ scores at $p<$ 0.01 level [28].

\section{Statistical analysis}

The statistical program PASW Statistics for Windows, Version 18.0 (SPSS Inc., Chicago, IL, USA) was used was data analysis. The general characteristics of the subjects were analyzed by descriptive statistics, and the pre- and post-intra-group changes were analyzed using the one-way ANOVA test. Statistical significance level of all data was $p<0.05$.

\section{Results}

There were not significant pre-intervention differences in flexion ROM, flexion strength, VAS, and SPADI in the HR-MWM group, MWM group, and control group ( $p>0.05$ ) (Table 1). The HR-MWM group showed statistically significant difference in the pre-post shoulder flexion ROM compared to the MWM group and the control group $(p<0.05)$, and the MWM group showed statistically significant differences in pre-post shoulder flexion ROM $(p<0.05)$. The HRMWM group showed a statistically significant improvement in muscle strength before and after the intervention compared to the MWM group and the control group $(p<0.05)$.

The HR-MWM group showed a statistically significant improvement in the VAS before and after the intervention compared to the MWM group and the control group. The HR-MWM group showed a statistically significant improvement in the SPADI results before and after the intervention compared to the MWM group and the control group $(p<0.05)$ (Table 2).

Table 1. Social and clinical demographics of the included participants

$(\mathrm{N}=45)$

\begin{tabular}{lcccc}
\hline \multicolumn{1}{c}{ Variable } & HR-MWM group $(\mathrm{n}=15)$ & MWM group $(\mathrm{n}=15)$ & Control group $(\mathrm{n}=15)$ & $x^{2 /} \mathrm{F}(p)$ \\
\hline Demographic and clinical data & & & & \\
Age $(\mathrm{y})$ & $56.73(7.72)$ & $53.93(6.79)$ & $56.80(8.24)$ & $41.400(0.738)$ \\
Height $(\mathrm{cm})$ & $164.00(7.18)$ & $163.53(5.12)$ & $165.13(7.93)$ & $39.743(0.392)$ \\
Weight $(\mathrm{kg})$ & $62.20(10.40)$ & $61.33(8.74)$ & $62.53(11.93)$ & $52.000(0.252)$ \\
ROM $\left({ }^{\circ}\right)$ & $123.67(5.50)$ & $124.33(3.20)$ & $123.33(4.08)$ & $0.204(0.816)$ \\
Flexion strength $(\mathrm{N})$ & $50.96(15.36)$ & $50.00(15.20)$ & $50.91(16.07)$ & $0.018(0.982)$ \\
VAS (mm) & $69.33(8.25)$ & $69.86(7.02)$ & $74.60(4.95)$ & $2.665(0.081)$ \\
SPADI (score) & $65.33(6.07)$ & $65.79(4.94)$ & $98.50(3.10)$ & $1.874(0.166)$ \\
\hline
\end{tabular}

Values are presented as mean (SD).

HR-MWM: hold relax+mobilization with movement, MWM: mobilization with movement, ROM: range of motion, VAS: Visual Analogue Scale, SPADI: Shoulder Pain And Disability Index. 
Table 2. Baseline scores of select outcomes

\begin{tabular}{|c|c|c|c|c|}
\hline & HR-MWM group $(n=15)$ & MWM group $(n=15)$ & Control group $(\mathrm{n}=15)$ & $\mathrm{F}(p)$ \\
\hline \multicolumn{5}{|c|}{ Shoulder flexion ROM $\left({ }^{\circ}\right)$} \\
\hline Before & $123.67(5.50)$ & $124.33(3.20)$ & $123.33(4.08)$ & $0.204(0.816)$ \\
\hline After & $166.67(4.88)$ & $162.00(2.54)$ & $152.67(3.20)$ & \\
\hline Amount of change & $43.00(4.55)^{\mathrm{a}, \mathrm{b}}$ & $37.67(4.95)^{\mathrm{a}}$ & $29.33(3.72)$ & $36.157(<0.001)$ \\
\hline \multicolumn{5}{|c|}{ Shoulder flexion strength $(\mathrm{N})$} \\
\hline Before & $50.96(15.36)$ & $50.00(15.20)$ & $50.92(16.07)$ & $0.018(0.982)$ \\
\hline After & $81.58(28.00)$ & $67.58(22.84)$ & $65.20(21.70)$ & \\
\hline Amount of change & $30.62(12.92)^{\mathrm{a}, \mathrm{b}}$ & $17.57(8.30)$ & $14.29(7.43)$ & $11.540(<0.001)$ \\
\hline \multicolumn{5}{|l|}{ VAS (mm) } \\
\hline Before & $69.33(8.25)$ & $69.87(7.02)$ & $74.60(4.95)$ & $2.665(0.081)$ \\
\hline After & $30.80(3.67)$ & $42.67(2.85)$ & $45.93(4.20)$ & \\
\hline Amount of change & $38.53(8.84)^{\mathrm{a}, \mathrm{b}}$ & $27.20(7.54)$ & $28.67(8.30)$ & $8.382(0.001)$ \\
\hline \multicolumn{5}{|l|}{ SPADI (score) } \\
\hline Before & $65.33(6.07)$ & $65.79(4.94)$ & $68.50(3.10)$ & $1.874(0.166)$ \\
\hline After & $31.69(3.36)$ & $34.66(2.90)$ & $38.35(2.96)$ & \\
\hline Amount of change & $33.64(7.19)^{\mathrm{a}, \mathrm{b}}$ & $22.87(6.04)$ & $23.06(5.95)$ & $13.885(<0.001)$ \\
\hline
\end{tabular}

Values are presented as mean (SD).

HR-MWM: hold relax+mobilization with movement, MWM: mobilization with movement, ROM: range of motion, VAS: Visual Analogue Scale, SPADI: Shoulder Pain And Disability Index.

${ }^{\text {a }}$ Statistically significant difference with control group $(p<0.05),{ }^{\text {b }}$ Statistically significant difference with MWM group $(p<0.05)$.

\section{Discussion}

In a previous study, Shah et al. [29] conducted a comparative study of ROM and pain by applying muscle energy technique and mobilization technique to patients with adhesive capsulitis surgery, and both the group applying mobilization technique and the group applying muscle energy techniques were effective I nshowing improvement.

The ROM of the group to which mobilization was applied showed greater improvement $(p<0.05)$. Decicco and Fisher [20] studied the effects of HRC and CRC techniques during PNF stretching on shoulder external rotation ROM in healthy athletes $(p<0.05)$.

Pragassme et al. [18] conducted a comparative study of pain intensity, shoulder abduction and external ROM in patients with adhesive capsulitis in subjects who were 40-60 years old, and the group that received the Mulligan technique showed significant differences in pain intensity and $\operatorname{ROM}(p<0.05)$.

In parallel with the HR technique, the MWM group had significantly greater shoulder flexion ROM than the group with only MWM applied ( $p<0.05)$, and the group with both HR technique and MWM alone had significantly greater shoulder flexion ROM than the control group $(p<0.05)$.

Decicco and Fisher [20] investigated the effects of using the HR technique on shoulder flexion and external rotation
ROM of the shoulder, and found increases in ROM with soft tissue elongation. In addition, Pragassme [18] studied the effects of MWM, which showed increases in ROM by reducing the stiffness of the joint sac secondary to arthroscopic surgery. It can be considered that shoulder flexion ROM increased with the HR technique and MWM.

In parallel with the HR technique, the MWM group had a significantly lower VAS than the MWM-only group ( $p<$ 0.05 ) and a significantly lower VAS than the control group $(p<0.05)$. The group applying only MWM was less significant than the control group. In parallel with the HR technique, the MWM group had significantly lower SPADI results than the MWM-only group $(p<0.05)$ and significantly lower SPADI results than the control group $(p<0.05)$.

The group applying only MWM was less significant than the control group. Although MWM was applied to improve muscle strength and reduce pain [10-13], this study did not show a significant difference from the control group. However, the reason why the MWM did not show a significant difference was that in the previous study, various MWMs such as shoulder flexion, extension, abduction, external rotation, and internal rotation were performed, but this study did not make a significant difference because only two types of MWMs were performed.

Handel et al. [30] explained that the HR technique increases the amount of efferent muscle contractions by in- 
creasing the elongation of soft tissues. This proved that the HR technique increased muscle strength than MWM [14]. SPADI is considered a useful clinical tool in pain assessment [28]. It is thought that the increase in ROM and the extension of soft tissues affect muscle strength, thus reducing the range of pain. This proved that the HR technique affected VAS and SPADI more than MWM [31,32].

This study showed that MWM combined with HR technique in patients with adhesive capsulitis had an effect on shoulder flexor muscle strength, VAS, and SPADI results during exercise. Through this, it is estimated that the intervention method of this study can be an effective method when applied to patients with adhesive capsulitis surgery in clinical practice.

However, the number of subjects in this study is small, so there are limitations. It cannot be ruled out that no other MWMs could be added that could be revealed by implementing two of the methods applied among the MWMs. In addition, it cannot be ruled out that no other HR techniques have been added, which may appear as two implementations of the HR technique. Therefore, future studies should take into consideration of these limitations when applying various methods of MWM to patients with adhesive capuslitis. It is considered that additional research is needed to address these limitations by applying various methods of HR technique, and further research is needed for rehabilitation of patients with adhesive capsulitis.

\section{Conflict of Interest}

The authors declared no potential conflicts of interest with respect to the authorship and/or publication of this article.

\section{References}

1. Neviaser AS, Hannafin JA. Adhesive capsulitis: a review of current treatment. Am J Sports Med 2010;38:2346-56.

2. Tasto JP, Elias DW. Adhesive capsulitis. Sports Med Arthrosc Rev 2007;15:216-21.

3. Le HV, Lee SJ, Nazarian A, Rodriguez EK. Adhesive capsulitis of the shoulder: review of pathophysiology and current clinical treatments. Shoulder Elbow 2017;9:75-84.

4. Guler-Uysal F, Kozanoglu E. Comparison of the early response to two methods of rehabilitation in adhesive capsulitis. Swiss Med Wkly 2004;134:353-8.

5. Mao CY, Jaw WC, Cheng HC. Frozen shoulder: correlation between the response to physical therapy and follow-up shoulder arthrography. Arch Phys Med Rehabil 1997;78:857-9.
6. Vermeulen HM, Rozing PM, Obermann WR, le Cessie S, Vliet Vlieland TP. Comparison of high-grade and low-grade mobilization techniques in the management of adhesive capsulitis of the shoulder: randomized controlled trial. Phys Ther 2006;86: 355-68.

7. Holloway GB, Schenk T, Williams GR, Ramsey ML, Iannotti JP. Arthroscopic capsular release for the treatment of refractory postoperative or post-fracture shoulder stiffness. J Bone Joint Surg Am 2001;83:1682-7.

8. Neviaser AS, Neviaser RJ. Adhesive capsulitis of the shoulder. J Am Acad Orthop Surg 2011;19:536-42.

9. Abbott JH. Mobilization with movement applied to the elbow affects shoulder range of movement in subjects with lateral epicondylalgia. Man Ther 2001;6:170-7.

10. Backstrom KM. Mobilization with movement as an adjunct intervention in a patient with complicated de Quervain's tenosynovitis: a case report. J Orthop Sports Phys Ther 2002;32:86-94; discussion 94-7.

11. Konstantinou K, Foster N, Rushton A, Baxter D. The use and reported effects of mobilization with movement techniques in low back pain management; a cross-sectional descriptive survey of physiotherapists in Britain. Man Ther 2002;7:206-14.

12. Paungmali A, O'Leary S, Souvlis T, Vicenzino B. Hypoalgesic and sympathoexcitatory effects of mobilization with movement for lateral epicondylalgia. Phys Ther 2003;83:374-83.

13. Doner G, Guven Z, Atalay A, Celiker R. Evalution of Mulligan's technique for adhesive capsulitis of the shoulder. J Rehabil Med 2013;45:87-91.

14. Sharman MJ, Cresswell AG, Riek S. Proprioceptive neuromuscular facilitation stretching: mechanisms and clinical implications. Sports Med 2006;36:929-39.

15. Alter MJ. Science of flexibility. 3rd ed. Champaign (IL): Human Kinetics; 2004.

16. Youdas JW, Haeflinger KM, Kreun MK, Holloway AM, Kramer $\mathrm{CM}$, Hollman JH. The efficacy of two modified proprioceptive neuromuscular facilitation stretching techniques in subjects with reduced hamstring muscle length. Physiother Theory Pract 2010;26:240-50.

17. Yang JL, Chang CW, Chen SY, Wang SF, Lin JJ. Mobilization techniques in subjects with frozen shoulder syndrome: randomized multiple-treatment trial. Phys Ther 2007;87:1307-15.

18. Pragassame SA, Kurup VM. Efficacy of limited treatment frequency of Mulligan's mobilization with movement for frozen shoulder. Indian J Physiother Occup Ther 2014;8:218-23.

19. Waddington G, Diong J, Adams R. Development of a hand dynamometer for the control of manually applied forces. J Manipulative Physiol Ther 2006;29:297-304.

20. Decicco PV, Fisher MM. The effects of proprioceptive neuromuscular facilitation stretching on shoulder range of motion in overhand athletes. J Sports Med Phys Fitness 2005;45:183-7.

21. Dundar U, Toktas H, Cakir T, Evcik D, Kavuncu V. Continuous passive motion provides good pain control in patients with adhesive capsulitis. Int J Rehabil Res 2009;32:193-8.

22. Crouch DL. Musculoskeletal biomechanical analysis of brachial plexus injury and treatment [Doctor dissertation]. Winston-Salem: Wake Forest University; 2014.

23. Baschung Pfister P, de Bruin ED, Sterkele I, Maurer B, de Bie RA, Knols RH. Manual muscle testing and hand-held dynamom- 
etry in people with inflammatory myopathy: an intra- and interrater reliability and validity study. PLoS One 2018;13:e0194531.

24. Peolsson A, Hedlund R, Öberg B. Intra- and inter-tester reliability and reference values for hand strength. J Rehabil Med 2001;33: 36-41.

25. Crichton N. Visual analogue scale (VAS). J Clin Nurs 2001;10: 706.

26. Bijur PE, Silver W, Gallagher EJ. Reliability of the visual analog scale for measurement of acute pain. Acad Emerg Med 2001;8: 1153-7.

27. Mawdsley RH, Hoy DK, Erwin PM. Criterion-related validity of the figure-of-eight method of measuring ankle edema. J Orthop Sports Phys Ther 2000;30:149-53.

28. Seo HD, Lee KW, Jung KS, Chung YJ. Reliability and validity of the Korean version of Shoulder Pain and Disability Index. J Spec Educ Rehabil Sci 2012;51:319-36.
29. Suri SA, Anand M. Comparative study on the effectiveness of Maitland mobilization technique versus muscle energy technique in treatment of shoulder adhesive capsulitis. Indian J Physiother Occup Ther 2013;7:1-6.

30. Handel M, Horstmann T, Dickhuth HH, Gülch RW. Effects of contract-relax stretching training on muscle performance in athletes. Eur J Appl Physiol Occup Physiol 1997;76:400-8.

31. Birinci T, Razak Ozdincler A, Altun S, Kural C. A structured exercise programme combined with proprioceptive neuromuscular facilitation stretching or static stretching in posttraumatic stiffness of the elbow: a randomized controlled trial. Clin Rehabil 2019;33:241-52.

32. Nakra N, Quddus N, Khan SA, Kumar S, Meena RL. Efficacy of proprioceptive neuromuscular facilitation on shoulder function in secondary shoulder impingement. Int J Ther Rehabil 2013;20: 450-8. 